\title{
Characterization and Evaluation of Sparse Array Transducers for Small Particle Detection
}

\author{
Xiaotong Li \\ Centre for Ultrasonic Engineering \\ University of Strathclyde \\ Glasgow, United Kingdom \\ xiaotong.li@strath.ac.uk
}

\author{
Anthony Gachagan \\ Centre for Ultrasonic Engineering \\ University of Strathclyde \\ Glasgow, United Kingdom \\ a.gachagan@strath.ac.uk
}

\author{
Paul Murray \\ Centre for Signal and Image Processing \\ University of Strathclyde \\ Glasgow, United Kingdom \\ paul.murray@strath.ac.uk
}

\begin{abstract}
In this paper, we present a log spiral array transducer which has been designed as a flexible alternative to conventional ultrasonic sensor technology. Two prototype transducers with our proposed array pattern have been fabricated, one using a fiber Composite Element Composite Array Transducer (CECAT) structure, the other using a conventional 13 composite $(\mathrm{C} 1-3)$. Three measurements including the impedance response, inter-element cross-talk and pulse-echo response have been acquired for both transducers to evaluate and compare their performance. The results show that the CECAT structure improves the transducer performance in all three measurements. The CECAT transducer has also been used to acquire ultrasonic Total Focusing Method (TFM) images from our tank-tube phantom (a tube submersed in water) containing artificial anomalies (solid particles in the range $1 \mathrm{~mm}-2 \mathrm{~mm}$ ) to synthesize our intended application of detecting anomalies through ultrasonic transcranial imaging. An image analysis algorithm combining image differencing and the Hough Transform has been designed to detect and estimate the size of these particles in the images. The particles inside the phantom as well as the tube have been successfully detected and dimensioned to $0.1 \mathrm{~mm}$ precision.
\end{abstract}

Keywords-transducer characterization, particle detection

\section{INTRODUCTION}

Transcranial ultrasound has been widely used in stroke diagnosis and treatment, both of which benefit from the noninvasiveness, portability and low expense of ultrasound devices [1]. Researchers have been investigating new devices to further improve the performance of transcranial ultrasound, such as developing headsets to hold the ultrasound transducer [2], using transducer arrays to achieve operator-independent treatment for patients [3], and employing 2D sparse array transducers to achieve better imaging capabilities [4].

In [5], we proposed an irregular sparse array pattern which could be integrated into a transcranial ultrasound system. This paper describes the fabrication, characterization, and evaluation of two prototype transducers using that array pattern [5]. One implementation uses a Composite Element Composite Array Transducer (CECAT) structure [6]. The other has been manufactured using a conventional 1-3 composite (C1-3). Both sensors are waterproofed as some experiments are conducted under water. A tank-tube phantom has been used to evaluate the transducers' performance. Images processed from the transducers have been used to develop an image processing algorithm to automatically detect and size the particles inside the tube.

\section{FABRICATION OF PROTOTYPE TRANSDUCERS}

An array pattern, as shown in Figure 1(a), has been designed in [5]. It consists of 79 elements with 78 elements equally distributed in 13 arms and one element in the center. Both array devices, the CECAT and the C1-3, have the same electrode pattern but have different piezoelectric microstructures. Figure 1(b) illustrates the construction of the prototype transducers. The manufacturing process of each prototype transducer consists of 5 steps:

1) Fabricating the active layer.

2) Applying electrical connections to achieve individual control of all array elements.

3) Packaging the active layer and electrical interconnections inside a housing.

4) Applying an appropriate matching layer.

5) Ensuring exit for external electrical cabling is waterproof.
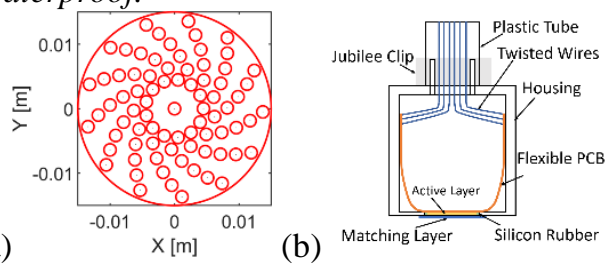

(b)

Fig. 1. (a) The 79-element log spiral array pattern used in manufacutring the prototype transducers. (b) Illustration of prototype transducer structure.

The main difference between the fiber CECAT and the C13 in terms of manufacturing happens when fabricating the active layer. The manufacturing process for the fiber CECAT active layer has been explained in [5]. It requires manual insertion of PZT fibers into a mould, while the 1-3 piezocomposite active layer uses the standard 'dice and fill' method [7]. To keep the consistency between the two array devices, the following design parameters are kept constant during fabrication.

1) PZT5A ceramic and CIBA-GEIGY CY221-HY956 epoxy are used as the active and passive materials.

2) The volume fraction is set at $50 \%$.

3) The layer thickness is set to $0.75 \mathrm{~mm}$.

4) Silver (Ag) is used as the material for electrodes $(\sim 500 \mathrm{~nm})$, with a thin layer ( 20nm) of chrome $(\mathrm{Cr})$ 
evaporated first to improve adhesion to the active material.

For the 1-3 composite active layer, the pillar width is set to $0.3 \mathrm{~mm}$ and the kerf width is set to $0.09 \mathrm{~mm}$. This gives around 29 pillars within each array element. This is very close to the number of fibers within each array element in the fiber CECAT active layer (28 fibers per element) as the volume fraction has been kept consistent for both devices. Images of the final active layers are shown in Figure 2.

(a)

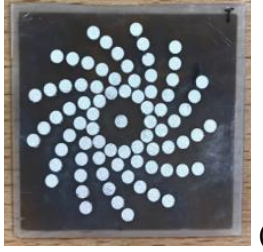

(b)

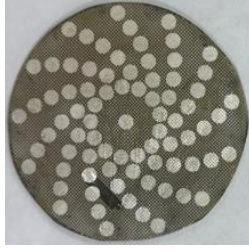

Fig. 2. The active layers using (a) the fiber CECAT structure and (b) the conventional 1-3 composite structure.

The electrical connection between the active layer and the external power source is achieved through two steps, first bonding the active layer to a flexible PCB using anisotropic conductive epoxy (Creative Materials Inc., USA) which is only conductive in the thickness direction, and then soldering twisted-pair wires to specific areas on the PCB. Twisted-pair wires are used to reduce noise between alternate signal channels within the array. The active layer, the flexible PCB, and the twisted-pair wires connected to the PCB are packaged inside a metallic housing, as illustrated in Fig. 1(b). A quarter wavelength matching layer made of $4 \%$ tungsten and CIBAGERIGY CY1301-HY1300 (hard set epoxy), selected from the CUE materials database [8], is applied on the prototype transducer to reduce the mismatching of acoustic impedance between the active layer and the load material (tissue or water). Finally, the length of twisted-pair wires, for external connection, is packaged inside a piece of plastic tubing. Silicon rubber and 5-min epoxy are used to seal the transducer and make it waterproof for testing using the tank-tube phantom.

\section{CHARACTERIZATION OF PROTOTYPE TRANSDUCERS}

\section{A. Impedance Response}

The electrical impedance response of all array elements has been measured before bonding the flexible PCB. The results are shown in Figure 3. The active layers were air loaded when performing the measurement to minimize damping to the array elements. Three parameters were measured and recorded based on the impedance response results: 1) the electrical resonance frequency $\left.\left(f_{e}\right), 2\right)$ the mechanical resonance frequency $\left(f_{m}\right)$, and 3 ) the coupling coefficient $\left(k_{t}\right)$. These parameters have been used to evaluate the uniformity for each active layer.

The averaged values for each parameter are listed in Table I. As shown in the table, the CECAT and the C1-3 have similar $f_{e}$, while the CECAT has higher $f_{m}$ than the $\mathrm{C} 1-3$. This situation is associated with the better performance of CECAT in terms of $k_{t}$ (0.64 to 0.59). For each parameter, a tolerance interval is created taking $\pm 5 \%$ of the mean value to evaluate the uniformity of the array elements. Importantly, $95 \%$ of the array elements in both transducers fall within this tolerance interval. The standard deviation results shown in Table I indicate that array elements in the CECAT perform better in terms of $f_{e}$ and $f_{m}$, while the array elements in the $\mathrm{C} 1-3$ has better uniformity in $k_{t}$.
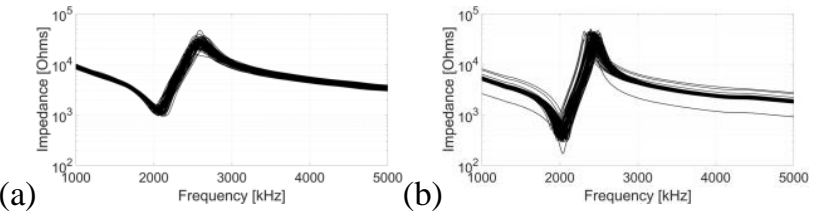

Fig. 3. Impedance response of all array elements for (left) the CECAT and (right) the C1-3.

TABLE I. ANALYZED PARAMETERS For BOTH ACTIVE LAYERS

\begin{tabular}{|l|c|c|c|c|c|c|}
\hline \multirow{2}{*}{ Device } & \multicolumn{2}{|c|}{$\boldsymbol{f}_{\boldsymbol{e}}$} & \multicolumn{2}{c|}{$\boldsymbol{f}_{\boldsymbol{m}}$} & \multicolumn{2}{c|}{$\boldsymbol{k}_{\boldsymbol{t}}$} \\
\cline { 2 - 7 } & Ave & Std & Ave & Std & Ave & Std \\
\hline CECAT & 2061 & 33.6 & 2587 & 43.5 & 0.64 & 0.014 \\
\hline C1-3 & 2030 & 39.4 & 2440 & 44.5 & 0.59 & 0.007 \\
\hline
\end{tabular}

\section{B. Inter-Element Cross-Talk}

The cross-talk between neighboring array elements has been measured using a 3D Laser Doppler Vibrometer (LDV) (Polytec Inc., Waldbronn, Germany). The measurement has been conducted with the active layer, the PCB, the twisted wires packaged in the housing and the matching layer attached. The experimental setup is shown in Figure 4(a). The transducer was placed on a platform with the front surface of the transducer in parallel to the laser source. The laser source was set to focus on the matching layer. Reflected laser signal was captured and analyzed by the LDV to evaluate the vibrational properties of the active layer. For each scan, only one array element was fired. Recall the log spiral array pattern; there are 13 arms with 6 elements in each arm, plus 1 element in the center. Thus, there are seven types of element neighboring situations in which mechanical cross-coupling should be investigated. Aiming to minimize scanning time, as opposed to scanning the entire active layer, only a region covering the fired element and its neighboring elements, as shown in Figure 4(b), was scanned.
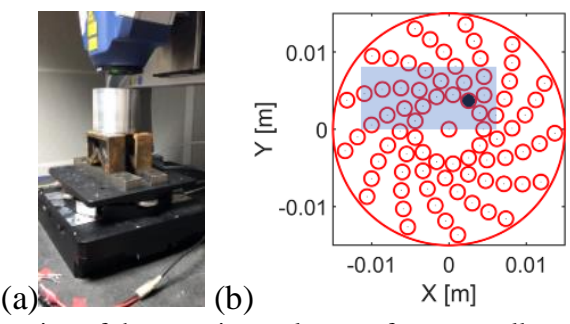

Fig. 4. (a) Illustration of the experimental set up for cross-talk measurement (b) Illustration of how the LDV scanning has been processed. The black circle represents the fired array element. The blue block represents the area that has been scanned.
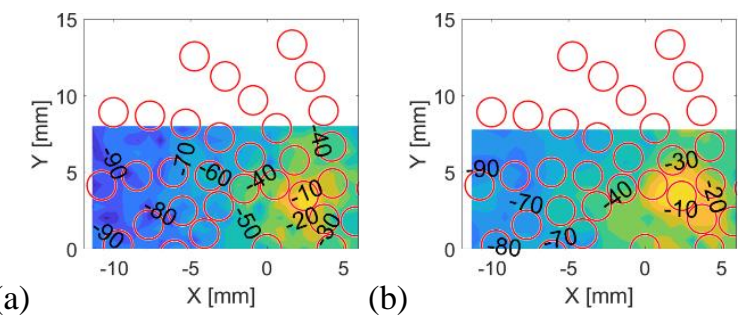

Fig. 5. Cross-talk contours for (a) the CECAT and (b) the C1-3 when firing the first element in the $3^{\text {rd }}$ arm individually. The red circles represent the theoretical position of the neighboring elements.

Within each arm, the array elements are numbered 1 to 7 from the center to edge. The cross-talk contours in $\mathrm{dB}$ for the 
first element within the $3^{\text {rd }}$ arm in the CECAT and C1-3 are shown in Figure 5. This situation is presented here since the array elements are closer in the center of the transducer than at the extremities and are thus of most interest for this experiment. As shown in Figure 5, the maximum cross-talk in the neighboring element is $-20 \mathrm{~dB}$ for the CECAT and $-10 \mathrm{~dB}$ for the C1-3. However, for both transducers, in all the element adjacent situations, the area with the maximum cross-talk corresponds to a small footprint.

\section{Pulse-Echo Response}

Figure 6 illustrates the setup for the pulse-echo test, which provides a characterization of the transducer sensitivity (amplitude), bandwidth and pulse-length. A glass block was placed at the bottom of a tank and used as a reflector for this experiment. The front surface of the transducer was positioned such that it was oriented in parallel to the glass block. The transducer was connected to a phased array controller, FIToolbox (Diagnostic Sonar Ltd, UK), through an adaptor to achieve individual control of each array element. A $2 \mathrm{MHz}, 80 \mathrm{~V}$ peak-to-peak, pulse was generated from the FIToolbox to drive each array element. The first received echo was recorded and analyzed to extract performance metrics for each transducer.

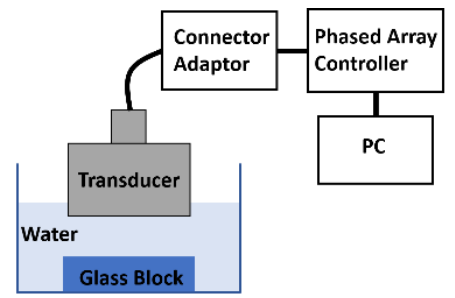

Fig. 6. Illustration of the experimental set up for the pulse-echo test.
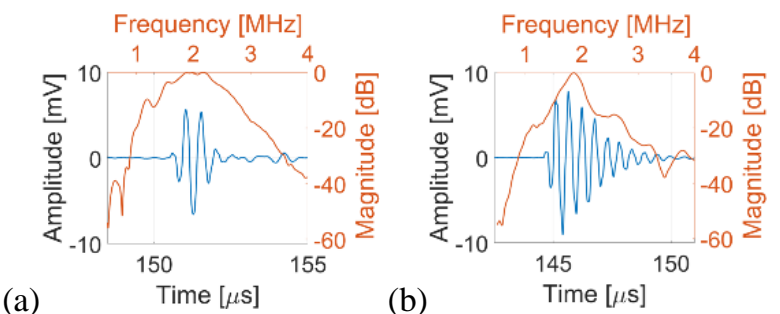

Fig. 7. Pulse echo response of the center element for (a) the CECAT and (b) the $\mathrm{C} 1-3$.

TABLE II. PULSE-ECHO RESPONSE RESUlts For Both PROTOTyPe TRANSDUCERS

\begin{tabular}{|l|c|c|c|c|}
\hline Device & $\begin{array}{c}\text { Center } \\
\text { Frequency } \\
{[\mathbf{M H z}]}\end{array}$ & $\begin{array}{c}\text { Pulse } \\
\text { Length } \\
{[\boldsymbol{\mu s}]}\end{array}$ & $\begin{array}{c}\text { Bandwidth } \\
{[\%]}\end{array}$ & $\begin{array}{c}\text { Peak-to-peak } \\
\text { Amplitude } \\
{[\mathbf{m V}]}\end{array}$ \\
\hline CECAT & 1.95 & 1.72 & 47.44 & 11.17 \\
\hline C1-3 & 1.85 & 2.67 & 30.95 & 16.67 \\
\hline
\end{tabular}

Pulse-echo responses were successfully acquired from all the 79 array elements from the $\mathrm{C} 1-3$, while 3 array elements from the CECAT failed to record a satisfactory response. The average values for each parameter are list in Table II. Figure 7 presents the example pulse-echo response and the corresponding spectrum of the center element from the CECAT and the C1-3. The center frequency is $1.95 \mathrm{MHz}$ for the CECAT and $1.85 \mathrm{MHz}$ for the $\mathrm{C} 1-3$. Both are slightly lower than the $2 \mathrm{MHz}$ desired working frequency. As shown in Figure 7, the CECAT has a shorter -20dB pulse length and broader bandwidth than the C1-
3, while the C1-3 has larger peak-to-peak amplitude. The results indicate that the CECAT has better spatial resolution, while the C1-3 performs better in terms of sensitivity. The differences in performance can be related to the difference of micro-structure between the active layers.

\section{Image Processing Algorithm To Detect PARTICLES INSIDE A TUBE}

In this Section, we present an image processing method which has been designed to identify solid particles that were introduced into our test vessels to simulate anomalies in blood flow. For our experiments, we have used a tank-tube phantom which is illustrated in Figure 8 and consists of a $3.5 \mathrm{~mm}$ (inner diameter) plastic tube which is submersed in water. The CECAT was placed above the tube and in parallel with the bottom of the tank. Ball bearings in range $1 \mathrm{~mm}-2 \mathrm{~mm}$ were placed at various positions inside the tube and within the area covered by the transducer aperture. The positions of the transducer and the tube remained fixed throughout the experimental process. The FIToolbox was used to control the transducer to collect data using the Full Matrix Capture (FMC) method [9] at a sampling frequency of $50 \mathrm{MHz}$. The FMC data was then processed using the Total Focusing Method (TFM) [9] to generate an image of the inspected area.

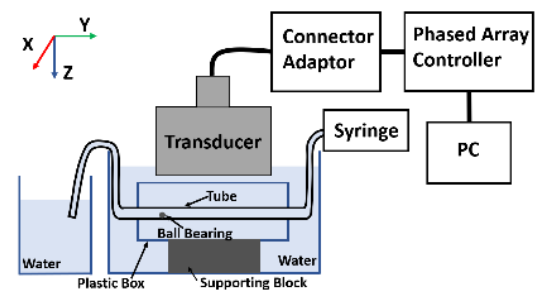

Fig. 8. Illustration of the experimental setup for imaging the tank-tube phantom using the CECAT transducer.

To simulate the concept of particles travelling through the imaging area, a series of TFM images starting with no particle and then with particles at different positions of the tube have also been captured. Figure 9 shows example TFM images captured using the CECAT. In Figure 9(a), the tube is empty, whereas in Figure 9(b) the tube contains three differently sized particles. Both images in Figure 9 show the decibel response at each location within range $-30 \mathrm{~dB}$ to $0 \mathrm{~dB}$. The four bright lines in Figure 9(a), from top to bottom, represent the top outer wall, the top inner wall, the bottom inner wall and the bottom outer wall of the tube separately. The true size of the particles in Figure 9(b), from left to right, is $1 \mathrm{~mm}, 1.5 \mathrm{~mm}$, and $2 \mathrm{~mm}$. As shown in Figure 9(b), all three particles have been successfully detected and appear as bright circles of different dimensions.

During image processing, all TFM images were converted into grayscale images (frames) with pixel intensity varying between 0 and 1 . The frame to be analyzed is defined as the Current Frame, $C F$. An imaging algorithm, as illustrated in Figure 10, was used to locate the particles and estimate their size. The first step is to define a Background Frame, $B F$, which should represent the motionless objects in the image (i.e. the tube) using:

$$
B F=\sum_{1}^{N} F(i) / N
$$


where $F(i)$ is the $i^{\text {th }}$ frame and, $N$ denotes the number of frames used in the calculation. In our experiments, $N=3$ (as only 3 images were captured). The Hough Transform is applied to $B F$ to detect the straight horizontal lines which represent the inner walls of the tube-phantom. Then, a Difference Frame, $D F$, is computed by calculating the absolute difference of $B F$ and $C F$. Ideally, when the object under inspection does not change, $D F$ should produce an empty image (all pixels $=0$ ). However, in practice, pixels intensities for the same inspected object can vary slightly through the sequence of frames which leads to noise in $D F$. In this imaging algorithm, the noise is reduced in two steps:

1) Any pixel with intensity lower than $-5 \mathrm{~dB}$ of the maximum intensity of the DF is set to 0 (black), while intensities of the remaining pixels are set to 1 (white).

2) A Morphological opening [10] is applied to the thresholded DF using a $5 \times 5$ disk mask Structuring Element (SE) to remove small clusters of noise.

Particles are detected in the resulting noise free $D F$ by locating non-zero pixel regions therein. Within each region, the pixel which coincides with the highest intensity pixel in the corresponding location in $C F$ is recorded as the particle position. Since the particles settle at the bottom of the tube, the distance from each particle to the bottom inner wall of the tube is used to estimate its size. Furthermore, the distance from the particle to both inner walls is used to estimate the tube's inner diameter.

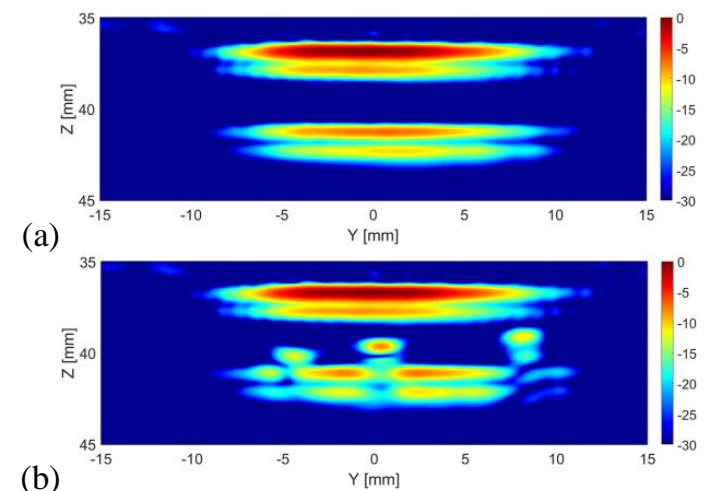

Fig. 9. Example TFM images from the CECAT trasnducer of the tank-tube phantom (a) with and (b) without particles inside the tube.

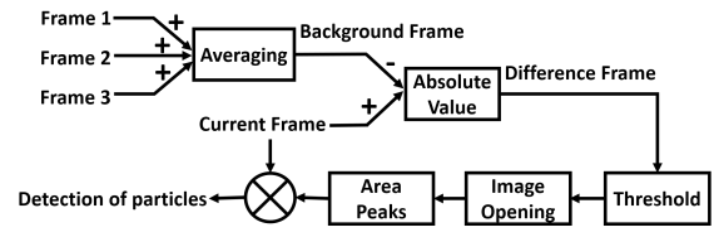

Fig. 10. Block diagram to illustrate the particle detecting algorithm.

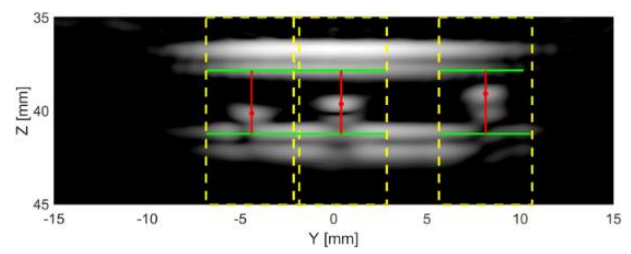

Fig. 11. An example Current Frame from the CECAT_79 with the particles detected. The dashed yellow lines indicate the regions in which a particle has been detected. The solid red lines represent the distance from the particles (red dots) to the top and bottom inner walls of the tube (green solid lines).
Figure 11 shows the results after applying the particle detection algorithm. All three particles have been successfully located. The estimated size is $1.1 \mathrm{~mm}$ for the $1 \mathrm{~mm}$ particle, $1.6 \mathrm{~mm}$ for the $1.5 \mathrm{~mm}$ particle, and $2.15 \mathrm{~mm}$ for the $2 \mathrm{~mm}$ particle. The estimated inner diameter of the tube is $3.4 \mathrm{~mm}$.

\section{CONCLUSION}

Two prototype transducers, a fiber CECAT and a conventional 1-3 composite have been manufactured through a similar process, with the main difference being the manufacturing method for the active layers. Results from the impedance response tests show that the utility of array elements is satisfactory for both active layers. However, the averaged $k_{t}$ for the fiber CECAT active layer is slightly higher than that of the piezocomposite active layers. Results from the LDV scanning show that the cross-talk level between neighboring array elements is lower overall for the fiber CECAT structure when compared to the C1-3. Pulse-echo tests show that the CECAT performs better in terms of pulse length and bandwidth, while the C1-3 demonstrates a higher sensitivity. An image processing algorithm for detecting and sizing small particles has been developed and evaluated using images acquired using the CECAT transducer to image a tank-tube phantom. The algorithm has shown appropriate accuracy when estimating the size of the particles and the tube. The next stage of this project will use phantoms made from tissue mimicking materials to evaluate the prototype transducer and image analysis algorithm.

\section{REFERENCES}

[1] R. Aaslid, T. M. Markwalder, and H. Nornes, "Non-invasive Transcranial Doppler Ultrasound Recording of Flow Velocity in Basal Cerebralarteries," Journal of Neurosurgery, vol. 57, pp. 769-774, 1982.

[2] B. Watt, "Design and Development of a Novel Transcranial Doppler Headset for Assessment of Cerebral Blood Flow", Master of Science, University of Nebraska-Lincoln, 2012.

[3] M. E. Schafer, J. Alleman, A. Alexandrov and K. Barlinn, "Development of an operator independent ultrasound therapeutic device for stroke treatment," 2012 IEEE International Ultrasonics Symposium, Dresden, 2012, pp. 1948-1951.

[4] B. Lindsey, E. Light, H. Nicoletto, E. Bennett, D. Laskowitz and S. Smith, "The ultrasound brain helmet: new transducers and volume registration for in vivo simultaneous multi-transducer 3-D transcranial imaging", IEEE Transactions on Ultrasonics, Ferroelectrics and Frequency Control, vol. 58, no. 6, pp. 1189-1202, 2011.

[5] X. Li, A. Gachagan, J. Dziewierz, R. O'Leary and P. Murray, "Design of a 2D sparse array transducer for integration into an ergonomic transcranial ultrasound system," 2017 IEEE International Ultrasonics Symposium (IUS), Washington, DC, 2017, pp. 1-4.

[6] G. Harvey, A. Gachagan, J. W. Mackersie, T. Mccunnie and R. Banks, "Flexible ultrasonic transducers incorporating piezoelectric fibres," in IEEE Transactions on Ultrasonics, Ferroelectrics, and Frequency Control, vol. 56, no. 9, pp. 1999-2009, September 2009.

[7] H. P. Savakus, K. A. Klicker, and R. E. Newnham, "PZT-epoxy piezoelectric transducers: A simplified fabrication procedure," Mater. Res. Bull., vol. 16, no. 6, pp. 677-680, Jun. 1981.

[8] R. L. O 'Leary, G. Hayward, G. Smillie, and A. C. S. Parr, "CUE Materials Database," Version 1., 2005.

[9] C. Holmes, B. Drinkwater and P. Wilcox, "Post-processing of the full matrix of ultrasonic transmit-receive array data for non-destructive evaluation", NDT \& E International, vol. 38, no. 8, pp. 701-711, 2005.

[10] C. Glasbey, Image analysis for the biological sciences. Chichester: John Wiley \& Sons, 1995. 